\title{
A child with three rare presentations of a common disease
}

\begin{abstract}
Autoimmune thyroiditis (Hashimoto thyroiditis) is the commonest thyroid disorder in childhood. It is associated with antibodies against thyroglobulin and thyroperoxidase with lymphocytic infiltration in the thyroid tissue. It may be associated with primary hypothyroidism, transient hyperthyroidism or an euthyroid state. It typically affects females-5 times more commonly than males. We aim to describe three rare presentations of a common disease (Hashimoto thyroiditis) in the same child and to discuss its clinical course and response to L-Thyroxin replacement during two years of treatment. We report an eleven years six months old female child, with severe long standing acquired primary hypothyroidism due to Hashimoto thyroiditis, who had, in addition to the typical hypothyroidism symptoms, three rare presentations at the same time including Hashimoto encephalopathy, gonadotropin independent precocious puberty and pituitary adenoma. She was treated with L-Thyroxin only with significant improvement and regression of her symptoms.
\end{abstract}

Keywords: thyroiditis, thyroperoxidase, encephalopathy, lymphocytic infiltration
Volume IO Issue I - 2020

\author{
Manal Mustafa Khadora,' Gururaj Aithalah,'² \\ Khaled El-Atawi, ${ }^{3}$ Mahmoud ElHalik ${ }^{4}$ \\ 'Pediatric Endocrinology Division, Department of Pediatrics, \\ UAE \\ ${ }^{2}$ Pediatric Neurology Division, Department of Pediatrics, UAE \\ ${ }^{3}$ Consultant Neonatologist, NICU, LWCH, UAE \\ ${ }^{4} \mathrm{Head}$ of Neonatal Intensive Care Unit, UAE
}

Correspondence: Manal Mustafa Khadora, Consultant Pediatric Endocrinologist, Pediatric Department, LWCH, UAE, Email MMKhadora@dha.gov.ae

Received: December 02, 2019 | Published: February 24, 2020
Abbreviations: CSF, cerebrospinal fluid analysis; MRI, magnetic resonance imaging; EEG, electroencephalography; HE, hashimoto encephalopathy

\section{Introduction}

Autoimmune Hashimoto thyroiditis is one of the most common thyroid diseases in childhood. It is associated with thyroglobulin and thyroperoxidase antibodies. It is characterized by lymphocytic infiltration in the thyroid tissue. It may be associated with primary hypothyroidism, transient hyperthyroidism or an euthyroid state. It typically presents in adolescents, affecting females 3-5 times more commonly than males. To screen for primary hypothyroidism, TSH should be measured. Elevated TSH mandate measuring the free T4 to differentiate overt (low free T4) from subclinical (normal free T4) hypothyroidism. The presence of adequate levels of thyroid hormone is very important to optimize neurological development and growth during childhood. The paediatrician should have a high index of suspicion to recognize the thyroid dysfunction in its early stages in order to avoid its serious complications.

\section{Objectives}

This report highlights 3 different rare presentations of Hashimoto thyroiditis in the same child. In addition, recovery from Hashimoto encephalopathy with only Thyroxin replacement therapy without the use of steroids raises additional therapeutic option in this disease.

\section{Case presentation}

Our patient is an eleven years six months old female child. She was born premature at 27 weeks gestation by emergency lower segment caesarean section (due to maternal pre-eclampsia) with birth weight of $680 \mathrm{gms}$. She stayed in neonatal intensive care unit for 3 months and had stormy postnatal course with occurrence of most of prematurity related complications. She had normal developmental milestones. She was in grade four in a mainstream school. Her speech was normal. She was not on any long-term medications. Her parents were nonconsanguineous. Her mother had Hashimoto thyroiditis in a euthyroid state, not on levothyroxin replacement. She had two other healthy younger siblings. No other significant family history of any endocrine disorders. Our patient presented to the emergency department at the age of 11 years 6 months with complaints of sudden onset of headache, increasing lethargy, rapidly progressive expressive aphasia, abnormal behavior, unsteady gait, and acute urinary incontinence for three days duration. She had been noticed by her parents to have static growth for the past two years prior to current presentation. A detailed history revealed that she had the typical symptoms of hypothyroidism, mainly constipation, cold intolerance, poor school performance in the recent past.

On clinical examination, she was walking with support, aphasic with dull course facies, dry skin and cold peripheries. No goiter was observed. Her height was $116 \mathrm{~cm}(-4.26 \mathrm{SD})$ and weight $23.9 \mathrm{~kg}(-3.0$ $\mathrm{SD})$. Increased upper: lower body ratio 1.1. Bradycardia, heart rate 60/ min with blood pressure $99 / 68 \mathrm{mmHg}$. Her neurological examination revealed truncal ataxia, increased tone in both lower limbs with brisk deep tendon reflexes and normal plantar reflexes. The cranial nerves were normal with no other cerebellar signs. Puberty staging: breast Tanner 3, absent pubic hair with sparse axillary hair.

\section{Initial investigations:}

a. Serum TSH > $150 \mathrm{mIU} / \mathrm{L}(0.7-4.6)$, FT4: $3.2 \mathrm{pmol} / 1$ (10.421.36), FT3 $0.7 \mathrm{pmol} / 1$ (3.5-7.3)

b. Anti thyroglobulin antibodies $494.5 \mathrm{IU} / \mathrm{ml}$ (negative < 100), and antithyroid peroxidase antibodies $1212 \mathrm{U} / \mathrm{ml}$ (negative < $50 \mathrm{U} / \mathrm{ml})$.

c. Serum prolactin $1620 \mathrm{mIU} / \mathrm{L}$ (102-496), repeated after 14 days $2402 \mathrm{mIU} / 1$. 
d. Random LH $0.2 \mathrm{mIU} / \mathrm{ml}$ (for breast Tanner III 0.3-4.12), FSH $7.7 \mathrm{mIU} / \mathrm{ml}$ (0.4-6.5) and serum estradiol $39 \mathrm{pmol} / 1$ (10-55).

e. ACTH am $42.2 \mathrm{pg} / \mathrm{ml}(<46)$, cortisol am 577nmol/1 (138-690).

f. IGF-1: 74.8ng/ml (111-551), IGF-BP3 1690ng/ml (2400-8400).

g. Electrocardiography: regular sinus bradycardia.

h. Bone age delayed; 8 years and 10 months at chronological age of 11 years 6 months (Figure 1).

i. Pelvis ultrasound: average size ovaries with multiple small follicles without ovarian cysts.

j. 1st brain MRI (at diagnosis): enlarged pituitary gland with superior expansion into the suprasellar cistern suggestive of pituitary adenoma, bilateral symmetric peri-ventricular white matter signal changes of previous leukomalacia related to prematurity (Figures 2-4)

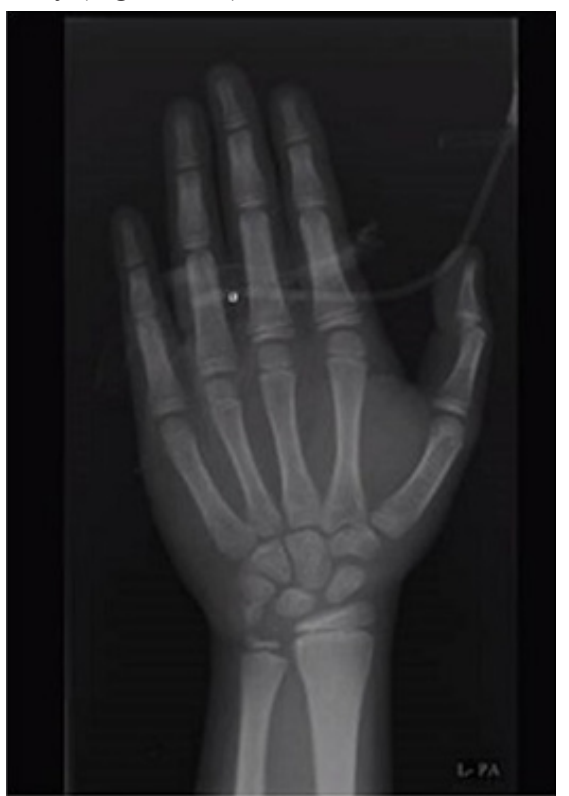

Figure I Bone age delayed (8years and 10 months at chronological age of Ilyrs 6 months).

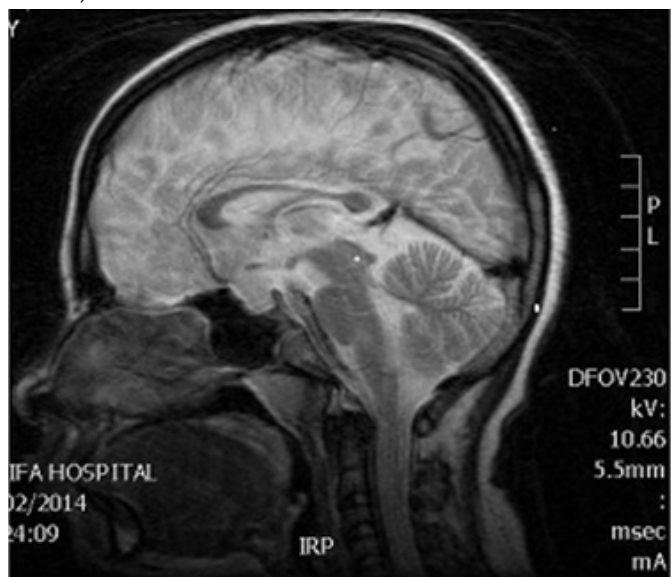

Figure 2 Brain MRI at diagnosis, sagittal view: enlarged pituitary gland (pituitary adenoma), bilateral symmetric peri-ventricular white matter signal changes of previous leukomalacia related to prematurity.

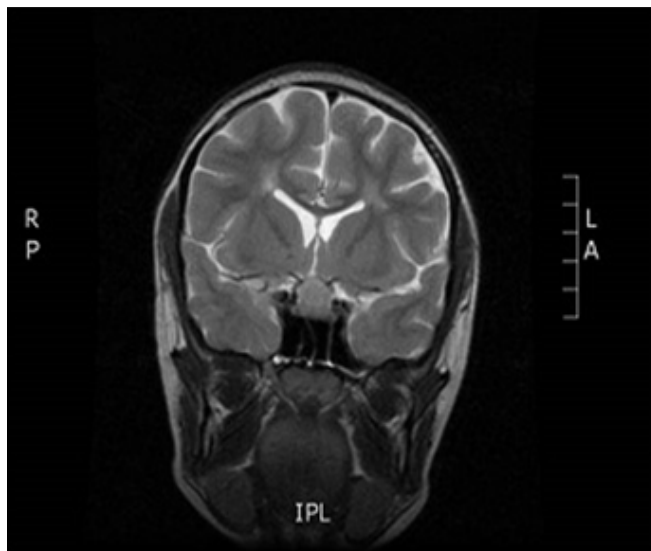

Figure 3 Brain MRI at diagnosis, coronal view.

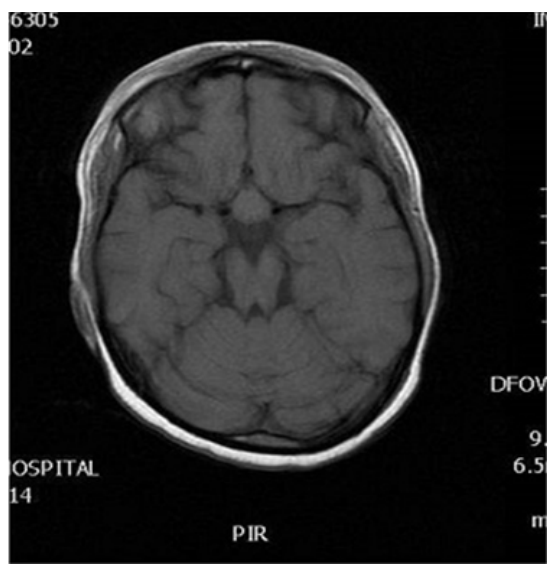

Figure 4 Brain MRI at diagnosis, axial view.

Her workup was consistent with severe auto immune primary hypothyroidism causing Hashimoto encephalopathy (after excluding other causes of acute encephalopathy). Hence, she was started on L-Thyroxin replacement, low dose initially $25 \mathrm{mcg}$ orally once daily, which was increased gradually to the full replacement dose of $75 \mathrm{mcg}$ orally once daily. She showed significant improvement in her hypothyroidism symptoms with complete resolution of the encephalopathic status and aphasia within 7days of starting Levothyroxine replacement only. She did not require any steroid treatment for her encephalopathy. The stigmas of hypothyroidism of dull coarse facies disappeared promptly and concomitantly following L-Thyroxin therapy. Her abnormal behavior and urinary incontinence resolved and her school performance improved.

After 8 weeks of starting L-Thyroxin treatment, all her laboratory investigations normalized. Her thyroid function showed euthyroid status, TSH 2.28mIU/L (0.7- 4.6), FT4: 17.3pmol/1 (10.4-21.36), Serum prolactin $399 \mathrm{mIU} / \mathrm{L}$ (102-496). Her pubertal signs remained same initially for the first 8 months following L-Thyroxine replacement, then her pubic hair grew progressively from tanner II to VI. After almost 12 months of starting L-Thyroxin, at the age of 12 years and six months, at breast Tanner VI, pubic hair tanner $\mathrm{VI}$, she got her first menses with random LH $8.6 \mathrm{mIU} / \mathrm{ml}$ (for breast Tanner VI 0.72-15.01), FSH $6.2 \mathrm{mIU} / \mathrm{ml}$ (1.26-7.37) and serum estradiol $75 \mathrm{pmol} / 1$ (25-345). Her repeated bone age was equal to twelve years. Her height improved to $132.4 \mathrm{~cm}(-3.45 \mathrm{SD})$ after 18 
months of L-Thyroxin replacement. Repeated IGF-1 improved to $277 \mathrm{ng} / \mathrm{ml}$ (111-551) and IGF-BP3 3520ng/ml (2400-8400). A follow up pituitary MRI showed significant reduction in size of her pituitary adenoma. It regressed to mildly enlarged pituitary gland (hyperplasia) (Figure 5). She remained euthyroid clinically and biochemically with regular menses throughout the follow up period.

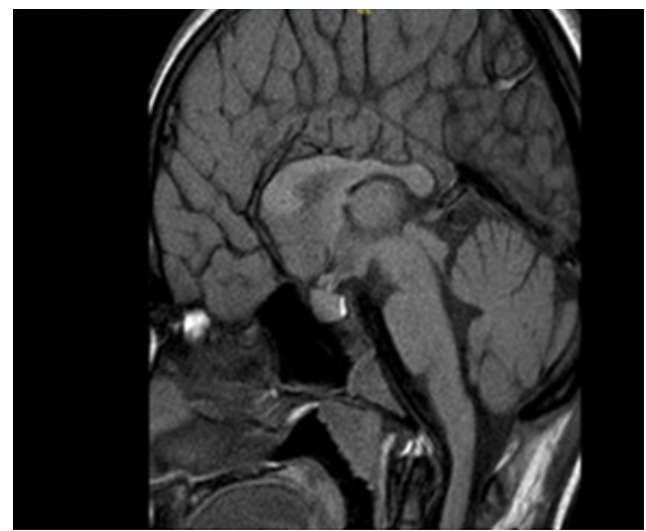

Figure 5 Brain MRI after one year of treatment, sagittal view: significant regression in size of pituitary adenoma to mild pituitary hyperplasia.

\section{Discussion}

Hashimoto thyroiditis, known as chronic lymphocytic thyroiditis or autoimmune thyroiditis, occurs mostly in females. It is the commonest cause of acquired primary hypothyroidism in children older than the age of 6 years in North America, affecting up to $1.2 \%$ of children at school age. ${ }^{1}$ The onset of acquired hypothyroidism during childhood is insidious with delayed diagnosis. Hypothyroid children are usually discovered because of the finding of a goiter or stunted growth, which might occur several years before establishing the diagnosis. Hypothyroid children are relatively overweight for height. ${ }^{1}$ Hashimoto's encephalopathy (HE) is a steroid-responsive, progressive or relapsing encephalopathy caused by high titers of serum anti-thyroid antibodies. It is often an under-diagnosed condition. It can be diagnosed if the patient has the following 3 findings: altered level of consciousness, or cognitive dysfunction without evidence of CSF viral or bacterial infection, and high serum titer of antithyroid peroxidase, or anti-thyroglobulin antibodies. ${ }^{2}$ Clinically, it usually starts insidiously, with symptoms of unexplained seizures, agitation, with or without other associated neurologic symptoms. Other neurological symptoms described are psychosis, behavioral changes, cognitive dysfunction, speech disturbance, cerebellar signs including ataxia, hallucination, involuntary movements, and coma. It can occur also in a relapsing-remitting manner of psychiatric illness and cognitive deterioration. ${ }^{2}$

Hashimoto's encephalopathy is well described in adults, but it is rarely diagnosed in children and adolescents. In literature there are only few pediatric case reports of this disease. In adults, a systematic review published in 2006 identified only 121 published cases of Hashimoto encephalopathy (HE). ${ }^{3}$

Interestingly, the occurrence of Hashimoto encephalopathy is not related to the patient's thyroid function status; but antithyroid antibodies are always present in the affected children. Anti thyroperoxidase antibodies positivity reaches $100 \%$, whereas anti thyroglobulin antibodies are less frequently positive. ${ }^{4}$ The pathogenesis of HE is not well understood, but the bulk of evidence suggests that it is an autoimmune vasculitis with immune complex deposition, possibly affecting the cerebral microvasculature. ${ }^{5}$ It is not due to thyroid disease as most of affected patients have normal thyroid function at the time of diagnosis. Unfortunately, this encephalopathy is underdiagnosed in children, so a high index of suspicion is important for early diagnosis. For that, screening for anti-thyroid antibodies should be performed in patients presenting with unexplained encephalopathy, particularly, if they have additional features of thyroid disease. The diagnosis of HE should be considered if anti-thyroid antibodies levels are elevated with no other explanation for encephalopathy. ${ }^{4}$ Other tests such as CSF (cerebrospinal fluid) analysis, MRI (magnetic resonance imaging), EEG (electroencephalography), and other laboratory investigations are essential in these patients to exclude other causes of encephalopathy but their findings are nonspecific in HE.

Corticosteroids along with the treatment of any concurrent hypothyroidism are the standard treatment of Hashimoto encephalopathy. Immunoglobulins and plasmapheresis are used for steroid-resistant patients. ${ }^{5}$ Most reported patients with Hashimoto encephalopathy improved after glucocorticoid treatment. In contrast, our reported patient improved significantly on thyroid hormone replacement only without using corticosteroids. Brain et al. ${ }^{7}$ in 1966 was the first to describe Hashimoto's encephalopathy. He reported a 49-year-old male with "Hashimoto's disease, encephalopathy and recurrent stroke-like episodes". ${ }^{6}$ This patient was treated with an anticoagulant and prednisone for 3 months without improvement. His neurologic symptoms improved with the use of levothyroxine only. The case conclusion was "Antibody studies in unexplained encephalopathy will show whether the described case is a syndrome or a coincidence." This reported case supports our finding that HE improved with Thyroxin replacement not with the use of corticosteroids.

In a recent retrospective observational study of 42 children with encephalitis manifested with acute neurological and behavioral symptoms by Nafissa et al, ${ }^{8}$ eight fulfilled the diagnostic criteria of HE. ${ }^{7}$ Five children had a relapse soon after the first episode (median 18 days (range 17-188)) despite steroid therapy. They all were treated with immunosuppressive therapy and two of them developed neurological sequelae by their last follow-up (after $4 \pm 1.3$ years). The prognosis of Hashimoto encephalopathy (HE) is generally good. Delayed diagnosis and treatment might be associated with incomplete recovery. ${ }^{8}$ Previous case reports suggested that most patients can improve with treatment even after years of diagnosis and some might have spontaneous recovery. ${ }^{8}$ Pituitary gland hyperplasia is usually seen in patients with severe long-standing primary hypothyroidism, due to thyrotrope and lactotrophs hyperplasia in response to hypersecretion of TRH. This pituitary hyperplasia can be significant since it mimics a prolactin-secreting tumor or pituitary macroadenoma. ${ }^{9}$ The diffuse pituitary enlargement associated with severe primary hypothyroidism is reversible with appropriate thyroid hormone treatment. ${ }^{10,11}$ In our reported patient, regression of her pituitary adenoma with levothyroxine treatment supports the hypothesis of pituitary hyperplasia secondary to primary hypothyroidism. Our case emphasize the importance of checking thyroid function tests in patients with hyperprolactinemia and/or pituitary adenoma. This helps to avoid unnecessary investigations and pituitary surgical intervention.

Children with hypothyroidism generally have delayed pubertal development. Rare association of precocious puberty and 
hypothyroidism may occur in almost $50 \%$ of children with severe long standing untreated patients. ${ }^{12}$ The main features of hypothyroidism induced pseudo precocious puberty include thelarche with or without galactorrhea, menarche in the absence of sexual hair and retardation of linear growth with delayed bone age, quite opposite of what is seen in patients with true precocious puberty. ${ }^{12}$ The underlying hormonal mechanisms in hypothyroidism- associated precocity remain obscure. Wyk and Grumbach tried to explain precocious puberty in long-standing severe hypothyroidism by an overlap in negative feedback regulation with overproduction of gonadotropins as well as thyrotropin (both share common $\alpha$ subunit) in response to thyroid deficiency. In boys, testicular enlargement may be inappropriate for the stage of puberty. ${ }^{13}$

Idiopathic intracranial hypertension (pseudotumor cerebri), a disorder of high intracranial pressure without focal neurologic dysfunction, may develop when children with severe hypothyroidism are treated with conventional doses of Levothyroxine. ${ }^{14}$ Thus treatment is initiated with one third to one half of the usual dose of Levothyroxine. After 2-4 weeks, the patient can be advanced to the conventional doses. However, children with profound hypothyroidism may develop pseudotumor cerebri even if treatment is initiated with low doses of levothyroxine. ${ }^{15,16}$

\section{Conclusion}

Hashimoto thyroiditis is the most common cause of acquired primary hypothyroidism in children. Patients with hypothyroidism do not always present with typical symptoms. Our case illustrates that primary hypothyroidism can present with rare presentations and pituitary mass. Hashimoto's encephalopathy is rarely suspected at presentation. Therefore, greater awareness of its signs by clinicians is necessary for proper diagnosis. This suggests the importance of thyroid function testing during the investigation of unexplained encephalopathy, pseudo precocious puberty, short stature, hyperprolactinemia and pituitary adenoma in order to avoid unnecessary diagnostic work-up and delayed diagnosis that might lead to serious complications. To the best of our knowledge, this is the first case report to describe the occurrence of three rare presentations in the same patient at initial presentation, and the first to describe the improvement of Hashimoto encephalopathy on Thyroxin replacement only.

\section{Acknowledgments}

None.

\section{Conflicts of interest}

The authors declare there are no conflicts of interest.

\section{References}

1. Foley TP: Disorders of the thyroid in children, in Sperling MA editor. Pediatric Endocrinology. Philadelphia, W.B. Saunders; 1996. p. 172 174.

2. Peschen-Rosin R, Schabet M, Dichgans J. Manifestation of Hashimoto's encephalopathy years before onset of thyroid disease. Eur Neurol. 1999;41(2):79-84.

3. Ferracci F, Bertiato G, Moretto G. Hashimoto's encephalopathy: epidemiologic data and pathogenetic considerations. J Neurol Sci. 2004;217(2):165-168.

4. Chong JY, Rowland LP, Utiger RD. Hashimoto Encephalopathy: Syndrome or Myth?. Arch Neurol. 2003;60(2):164-171.

5. Ferracci F, Carnevale A. The neurological disorder associated with thyroid autoimmunity. J Neurol. 2006;253(8):975-984.

6. Kothbauer-Margreiter I, Sturzenegger M, Komor J, et al. Encephalopathy associated with Hashimoto thyroiditis: diagnosis and treatment. $J$ Neurol. 1996;243(8):585-593.

7. Brain L, Jellinek E, HBall K, Hashimoto's disease and encephalopathy. Lancet. 1966;2(7462):512-514.

8. Mamoudjy, Nafissa, Christian Korff, Hélène Maurey, et al Hashimoto's encephalopathy: Identification and long-term outcome in children. European Journal of Paediatric Neurology. 2013;17(3);280-287.

9. GayatriNA, Whitehouse WP. Pilot survey of Hashimoto's encephalopathy in children. Dev Med Child Neurol. 2005;47(8):556-558.

10. Passeri E, Tufano A. Large pituitary hyperplasia in severe primary hypothyroidism. J Clin Endocrinol Metab. 2011;96(1):22-23.

11. Alves C, Alves AC. Primary hypothyroidism in a child simulating a prolactin secreting adenoma. Childs Nerv Syst. 2008;24(12):1505-1508.

12. Coleen Adams MB. Primary hypothyroidism with intracranial hypertension and pituitary hyperplasia. Pediatric Neurology. 1994;10(2):166-168.

13. Garibaldi L. "Disorders of Pubertal development," in Nelson Text Book of Pediatrics, Kliegman RM, Behrman RE, Jenson HB, editors. 18th ed. New Delhi, India: Elsevier; 2007;2:2309-2316.

14. Wyk JJV, Grumbach MM. Syndrome of precocious menstruation and galactorrhea in juvenile hypothyroidism: an example of hormonal overlap in pituitary feedback. The Journal of Pediatrics. 1960;57(3):416-435.

15. Huseman CA, Torkelson RD. Pseudotumor Cerebri Following Treatment of Hypothalamic and Primary Hypothyroidism. Am J Dis Child. 1984;138(10):927-931.

16. Slyper A, Swenerton P. Experience with low-dose replacement therapy in the initial management of severe pediatric acquired primary hypothyroidism. Journal of Pediatric. 2011.

\section{Funding}

None. 